\title{
Complicações do cateter port a cath: subsídios para os cuidados de enfermagem
}

\section{Complications of port a cath catheter: subsidies for nursing care}

\author{
Rafaela Martins de Almeida Peixoto' • Sônia Regina de Souza ${ }^{2}$ Juliana da Costa Silva ${ }^{3}$ \\ Eveline Mendonça da Silva Mendes ${ }^{4}$ Denise de Assis Corrêa Sória ${ }^{5} \bullet$ Lívia Machado Fontes $^{6}$
}

\section{RESUMO}

O Sistema Único de Saúde deve estar orientado e capacitado para a atenção integral à saúde do paciente oncológico. Sendo assim, houve o despertar em apreender as complicações clínicas que levavam à retirada do cateter port a cath. Traçamos como objetivos: descrever as complicações que levam a retirada do cateter port a cath no paciente oncológico; analisar as complicações que levam à retirada do cateter port a cath no paciente oncológico, trazendo subsídios para os cuidados de enfermagem. $O$ método apresenta-se como pesquisa documental, de caráter quantitativo e natureza descritiva e retrospectiva de dados extraídos de prontuários, através de um instrumento de coleta de dados. No desdobramento dos achados das complicações relatadas referentes ao cateter port a cath, empregou-se a análise de estatística descritiva e a infecção foi a principal complicação. Conclui-se que o impacto do resultado da pesquisa poderá promover a melhoria da assistência, maior disponibilidade para as mudanças de atitude e comportamento da equipe de enfermagem.

Palavras-chave: Cateteres Venosos Centrais; Infecções Relacionadas a Cateter; Enfermagem Oncológica.

\section{ABSTRACT}

The Unified Health System (SUS) should be oriented and trained to provide comprehensive health care for cancer patients. Thus, there was an awakening in learning about the clinical complications that led to the removal of the catheter port a cath. The goals of this research are: to describe and analyze the complications that lead to the removal of the port to cath catheter in cancer patients, bringing subsidies to the nursing care. Documentary, quantitative and descriptive research of data extracted from medical records through an instrument of data collection. In the unfolding of the findings of the reported complications related to the port a cath catheter, the analysis of descriptive statistics was used, and infection was the main complication. The impact of the research result may promote improved care, greater availability for changes in attitude and behavior of the nursing team.

Keywords: Central Venous Catheters; Catheter-Related Infections; Cancer Nursing.

'Enfermeira especialista em Clínica e Cirúrgica. Universidade Federal do Estado do Rio de Janeiro. E-mail: rafaaela_martiins@yahoo.com.br.Autor correspondente;

${ }^{2}$ Doutora em Enfermagem. Universidade Federal do Rio de Janeiro. Professora Associada nível II. Diretora da Escola de Enfermagem Alfredo Pinto. Universidade Federal do Estado do Rio de Janeiro. E-mail: soniasilvio0@gmail.com;

3Doutoranda em Enfermagem. Universidade Federal Fluminense. E-mail: ju9costa@gmail.com;

${ }^{4}$ Enfermeira especialista em cuidados Oncológicos. Universidade Estácio de Sá. E-mail: evelineenf@gmail.com;

${ }^{5}$ Doutora em Enfermagem. Universidade Federal do Rio de Janeiro. Professora Associada nível IV. Chefe do Departamento de Enfermagem Médico-Cirúrgica. Escola de Enfermagem Alfredo Pinto. Universidade Federal do Estado do Rio de Janeiro. E-mail: denisesoria@terra.com.br;

${ }^{6}$ Enfermeira especialista em Clínica e Cirúrgica. Universidade Federal do Estado do Rio de Janeiro. E-mail: liviamfa@hotmail.com 


\section{INTRODUÇÃO}

O cateter port a cath é um dispositivo de borracha siliconizada, cuja extremidade distal se acopla a uma câmera puncionável, que permanece sob a pele, embutida em uma loja no tecido subcutâneo da região torácica, sobre uma superfície óssea. É implantado por meio de procedimento cirúrgico e costuma ter boa aceitação pelos pacientes por não requerer cuidados domiciliares e ter mínima interferência na auto-imagem, pois o dispositivo não se exterioriza, porém, exige-se ainda a punção percutânea ${ }^{(1)}$.

Esse cateter, além de oferecer maior conforto funcional, apresenta menor índice de infecção, quando comparado a outros cateteres disponíveis. Esteticamente, considera-se ideal para pacientes que requerem uso de quimioterapia sistêmica intermitente e prolongada(2). O cateter totalmente implantável minimiza $\circ$ risco de trombose, fácil punção, permite tratamento ambulatorial, é radiopaco, não interfere nas atividades diárias do paciente e preserva o sistema venoso periférico(3).

As indicações para o implante do cateter totalmente implantado são: administração de quimioterapia, hemoderivados, antibióticos, nutrição parenteral, analgésicos e necessidade frequente de coleta de amostra de sangue. Atualmente, cada vez mais pacientes oncológicos e hematológicos fazem uso deste tipo de dispositivo(4).

Apesar da grande utilidade desses cateteres, sua inserção e manutenção não são isentas de complicações ${ }^{(5)}$. Por permitirem uma utilização prolongada, facilitam também a ocorrência de complicações durante este período, como infecção, extravasamento, obstrução, trombose, hematomas e deslocamento do cateter ${ }^{(6)}$. Apresentam complicações diversas relacionadas ao seu implante, à manipulação e à manutenção. $A$ infecção em cateteres de longa permanência constitui complicação de grande morbimortalidade, com riscos e agravos adicionais em pacientes muitas vezes debilitados ou imunossuprimidos, como aqueles submetidos à quimioterapia $^{(7)}$. A prática de higienização nos serviços de saúde vem sendo amplamente estudada pelo seu comprovado benefício para redução das taxas de infecções associadas a assistência à saúde, no entanto, a baixa adesão à esta medida continua sendo um problema alarmante( ${ }^{(8)}$.

Estima-se para o Brasil no biênio 2018-2019, a ocorrência de 600 mil casos novos de câncer, para cada ano(9). Sendo assim, diante do número significativo de casos incidentes de câncer, o SUS deve estar orientado e capacitado para a atenção integral à saúde do paciente oncológico, numa perspectiva que contemple a promoção da saúde, as necessidades de saúde da população com câncer, o controle de complicações mais prevalentes nesse grupo e a garantia do direito à saúde.
Diante do exposto, delineamos como objeto de estudo as complicações do cateter port a cath. Com a finalidade de obter os resultados, traçamos as seguintes questões norteadoras: Quais são as complicações que levam à retirada do cateter port a cath no paciente oncológico?; Qual é o papel da enfermagem diante das complicações que levam à retirada do cateter port a cath? Para o alcance do objeto de estudo, definimos os seguintes objetivos: descrever as complicações que levam à retirada do tal cateter no paciente oncológico; analisar as complicações que levam à essa retirada, trazendo subsídios para os cuidados de enfermagem.

\section{MÉTODO}

Optou-se pela pesquisa documental e retrospectiva em prontuários, por descrever os fenômenos, e de se estabelecer correlações ou verificar a frequência probabilística de sua ocorrência ${ }^{(10)}$.

Realiza-se o estudo retrospectivo a partir de registros do passado, e é seguido a partir daquele momento até o presente ${ }^{(10)}$. É fundamental que haja credibilidade nos dados de registros a serem computados, em relação à exposição do fator e/ou à sua intensidade ${ }^{(I I)}$.

O método apresenta-se como pesquisa documental, de caráter quantitativo e natureza descritiva e retrospectiva de dados extraídos de prontuários, através de um instrumento de coleta de dados, cuja finalidade é descrever um fenômeno ou situação, mediante um estudo realizado em determinado espaço-tempo ${ }^{(12)}$. Utilizamos os conceitos teóricos de Minayo, que trabalha com o universo de significados, motivos, aspirações, crenças, valores e atitudes, o que corresponde a um espaço mais profundo das relações, dos processos e dos fenômenos que não podem ser reduzidos à operacionalização de variáveis ${ }^{(13)}$.

O estudo foi realizado no Hospital Federal de Bonsucesso, referência para tratamento oncológico. As questões éticas foram atendidas de acordo com a Resolução n. ${ }^{\circ} 466 / 2012$ do Conselho Nacional de Saúde. Para atender e cumprir as normas éticas determinadas por esta resolução, o referido estudo foi submetido aos procedimentos normativos do CEP UNIRIO sob o número do CAAE 570326I6.2.0000.5285 e número do Parecer I.700.76I e CEP do Hospital Federal de Bonsucesso sob o número do CAAE 57032616.2.300I.5253 e número do parecer I.764.235, configurando aprovação dos pareceristas destes Comitês.

Critérios de inclusão: ter implantado um dispositivo totalmente implantado entre 2014 e 2016 e pessoas que estão ou não em tratamento atual (no momento da coleta de dados). Critérios de exclusão: pacientes fora do espaço temporal citado acima e que não tenham em seu prontuário do Arquivo Médico o Boletim Operatório de implantação de cateter port a cath. 
A partir da emissão da listagem das cirurgias de implantação de cateter port a cath nos anos de 20I4, 2015 e 2016 pela secretaria do Centro Cirúrgico, encontrouse 93 pacientes. Foi analisado também o mapa diário do setor de quimioterapia dentro do espaço temporal da pesquisa e foram encontradas 92 implantações de cateter port a cath. Sendo assim, obtiveram-se um total de 185 pacientes com colocação do cateter. Após a verificação dos 185 prontuários, apenas 137 atenderam aos critérios de inclusão da pesquisa: 38 implantações de cateter port a cath no ano de 20I4; 46 implantações no ano de 2015; 53 implantações no ano de 2016.

Logo, para estes 137 prontuários aplicou-se o instrumento de coleta de dados, através da leitura completa de cada prontuário, sustentando a temática proposta e respondendo aos objetivos da pesquisa. Para a identificação dos prontuários na pesquisa, estabeleceu-se uma codificação dos instrumentos em uma sequência alfanumérica, onde a letra " $A$ " corresponde ao ano de 2014, a letra "B" ao ano de 2015 e a letra "C" ao ano de 2016; e após a organização dos anos, a ordem dos instrumentos foi estabelecida por um numeral. Para a identificação das quatro clínicas cirúrgicas que realizaram a implantação dos cateteres port a cath, utilizou-se a sequência alfa: "W";"X";"Y"; e "Z".

O Setor de Oncologia possui prontuário exclusivo de cada paciente, independente do prontuário já existente no Arquivo Médico. De uma busca de 137 prontuários do Serviço de Oncologia, apenas III foram encontrados nos Arquivos da Secretaria do Setor de Oncologia, e 26 prontuários não foram localizados (A2,A9,A 14,A 16,AI7,A25,A27, $A 29, A 30, A 37, B 1, B 3, B 15, B 18, B 19, B 20, B 27, B 28, C 3, C 4$, $C 17, C 20, C 23, C 26, C 32, C 40)$. Foi realizada a leitura e análise dos prontuários encontrados na Oncologia, e as informações pertinentes a pesquisa foram complementares ao instrumento de coleta de dados respectivo a cada paciente.

Em seguida, para facilitar e compreender a análise, os dados foram distribuídos em um quadro no Programa Microsoft Excel 2010 de acordo com todas as variáveis inseridas no instrumento de coleta de dados.

Para se obter os resultados, empregou-se a análise de estatística descritiva, que tem como objetivo a descrição dos dados, sejam eles de uma amostra ou de uma população. Pode incluir: verificação da representatividade ou da falta de dados; ordenação dos dados; compilação dos dados em tabela; criação de gráficos com os dados; calcular valores de sumário, tais como médias; obter relações funcionais entre variáveis ${ }^{(14)}$.

Para o embasamento desta pesquisa para análise e discussão dos resultados, aplicou-se a produção científica de artigos que abordam a temática da assistência oncológica, publicada nas bases de dados da BVS, dissertações e teses, entre outras.

\section{RESULTADOS}

Em relação ao sexo, durante o espaço temporal da pesquisa, $58,4 \%(n=80)$ das implantações de cateter port a cath foram realizadas em mulheres, enquanto $41,6 \%$ $(n=57)$ das implantações foram realizadas em homens. Os resultados mostraram uma faixa etária predominantemente adulta, evidenciando o número de idosos. Houve maior prevalência na faixa etária de 56 a 60 anos, representando $18,9 \%(n=26)$.

Em relação as clínicas cirúrgicas que realizaram as implantações de cateter port a cath, a Clínica "W" com 49,6\% $(n=68)$ foi a Clínica Cirúrgica que mais realizou implantações durante o espaço temporal da pesquisa, sendo em 2016 o ano em que mais houve implantaçãoes com 38,7\% ( $n=53)$. Como diagnóstico para cada paciente, a análise da pesquisa evidenciou 19 tipos de câncer, sendo o câncer de cólon com prevalência de $37,2 \%(n=5 I)$. No entanto, $63(46 \%)$ pacientes com apresentação de metástase, sendo importante ressaltar a ocorrência em fígado $(n=4 I)$ e pulmão $(n=16)$. Diante das patologias pré-existentes à implantação do cateter port a cath, 73 (53,3\%) informaram positivamente, evidenciando Hipertensão Arterial Sistólica $(n=58)$ e Diabetes Mellitus ( $n=23)$.

As indicações clínicas "Quimioterapia neoadjuvante/ adjuvante" $(n=15)$ e "Falência de acesso vascular periférico" $(n=I)$ foram as mais relatadas. Com relação à prevalência da data de implantação, em 2014 foram realizadas $27,6 \%(n=38)$ de implantações; em 2015 foram realizadas $33,4 \%(n=46)$; em 2016 foram realizadas $38,6 \%(n=53)$.

Para as implantações do cateter foram informadas duas veias: subclávia e jugular interna.A veia subclávia foi a puncionada em 122 pacientes, representando $89 \%$ das implantações, sendo 78,8\% $(n=108)$ com localização em subclávia direita, 9,5\% $(n=13)$ em subclávia esquerda e $0,7(n=I)$ sem especificação de qual subclávia. Enquanto a veia jugular interna foi majoritariamente de localização à direita, com 7,3\% $(n=10)$. Em 3,6\% $(n=5)$ dos prontuários não foram informados a veia de implantação de cateter port a cath. Para todas as 137 implantações de cateter port a cath, realizadas no centro cirúrgico, foram administradas anestesia local.

Das I 37 implantações, 28 (20,4\%) apresentaram complicações clínicas, conforme Figura I, sendo 17,8\% ( $n=5)$ no ano de $2014,50 \%(n=14)$ no ano de 2015 e $32,1 \%$ $(n=9)$ no ano de 2016. Quanto à Clínica Cirúrgica, 42,8\% $(n=12)$ das implantações foram realizadas pela Clínica "W", 53,6\% ( $n=15)$ das implantações foram realizadas pela Clínica "X" e 3,5\% $(n=I)$ das implantações foram realizadas pela Clínica " $Z$ ".

Conforme a Figura 2, diante das complicações clínicas encontradas durante a análise da pesquisa, foi possível descrever as respectivas condutas assistenciais. 
FIGURA 1 - Apresentação das complicações clínicas diante das implantações de cateter port a cath. Hospital Federal de Bonsucesso, Rio de Janeiro, 2018

\section{Complicações apresentadas}

$\mathbf{n}$

Infecção do cateter 14

Disposicionamento de cateter

Apresentação de quadro clínico com febre

Ausência de fluxo e refluxo ao puncionar cateter

Pneumotórax

Ausência de progressão na implantação do cateter

Abaulamento em região infraclavicular

Extravasamento de quimioterapia

Trombose

FIGURA 2 - Apresentação das complicações clínicas diante das implantações de cateter port a cath com suas respectivas condutas assistencias e execuções referenciadas. Hospital Federal de Bonsucesso, Rio de Janeiro, 2018.

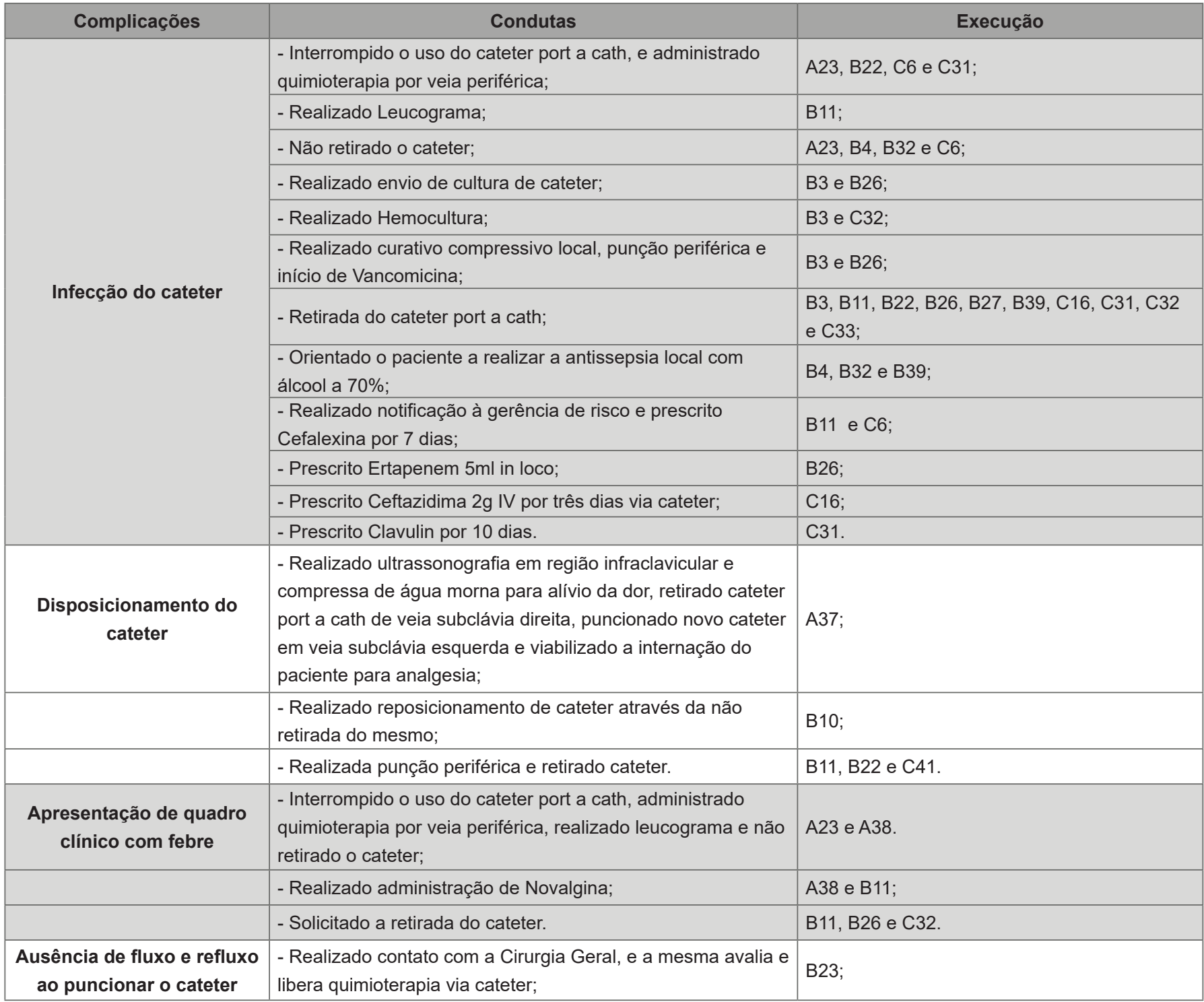




\begin{tabular}{|c|l|l|}
\hline & $\begin{array}{l}\text { - Suspenso quimioterapia, realizado Raio-X de tórax e } \\
\text { retirado cateter. }\end{array}$ & C29. \\
\hline Pneumotórax & $\begin{array}{l}\text { - Solicitado internação hospitalar e tomografia } \\
\text { computadorizada de tórax, realizada drenagem de tórax em } \\
\text { selo d'água. Posteriormente, cateter port a cath fluindo e } \\
\text { refluindo, liberado quimioterapia. }\end{array}$ & C1 e C27. \\
\hline $\begin{array}{c}\text { Ausência de progressão na } \\
\text { implantação do cateter }\end{array}$ & $\begin{array}{l}\text { - Retirado cateter de veia subclávia direita e realizada } \\
\text { punção com sucesso em veia jugular interna direita; }\end{array}$ & B14 e B15. \\
\hline $\begin{array}{c}\text { Abaulamento em região } \\
\text { infraclavicular }\end{array}$ & $\begin{array}{l}\text { - Interrompida quimioterapia e paciente encaminhado para } \\
\text { Cirurgia Geral; }\end{array}$ & A15. \\
\hline $\begin{array}{c}\text { Extravasamento de } \\
\text { quimioterapia }\end{array}$ & $\begin{array}{l}\text { - Retirado cateter port a cath de veia subclávia direita, } \\
\text { puncionado novo cateter em veia subclávia esquerda e } \\
\text { viabilizado internação hospitalar para analgesia; }\end{array}$ & A37. \\
\hline Trombose & - Liberado quimioterapia pela Cirurgia Geral. & B23. \\
\hline & - Solicitada retirada de cateter port a cath. & B36. \\
\hline
\end{tabular}

Contudo, não foram encontrados registros em prontuários de condutas assistenciais em A34 e BI3.

Após apresentar complicações e realizado condutas pertinentes, apenas $53,6 \%(n=15)$ dos cateteres foram retirados. Foi observado e analisado o tempo (em dias) com o cateter port a cath da implantação até a sua retirada.Apenas um prontuário não informou a data de retirada, impossibilitando a contagem dos dias dessa variável. O menor tempo com o cateter da implantação até a sua retirada foi observado em "BI4" (I dia), já o maior tempo foi averiguado em "B26" (480 dias). Sendo assim, foi possível encontrar uma média de tempo de 99 dias com o cateter port a cath da implantação até a sua retirada por complicações.

Neste estudo foram constatados 29 óbitos dos 137 pacientes com implantação de cateter port a cath de 2014 a 2016. Das 38 implantações realizadas no ano de 2014 , constatou-se II (28,9\%) óbitos nesse grupo de pacientes, com uma média de tempo de 273 dias da implantação até o óbito; das 46 implantações realizadas no ano de 2015 , constatou-se 16 (34,7\%) óbitos nesse respectivo grupo de pacientes, com uma média de tempo de 176 dias da implantação até o óbito; e das 53 implantações realizadas no ano de 2016 , constatou-se $2(3,7 \%)$ óbitos nesse respectivo grupo, com uma média de tempo de 182 dias da implantação até o óbito. De acordo com a Figura 3, a partir da análise dos três anos (2014 a 2016), o menor tempo com o cateter da implantação até o óbito foi observado em "BI2" (55 dias), e o maior tempo foi averiguado em "A6" (605 dias). Sendo assim, foi possível encontrar uma média de tempo de 213 dias com o cateter port a cath da implantação até o óbito.

\section{DISCUSSÃO}

Diante dos resultados, buscaram-se evidências na literatura para desenvolver um aporte científico para as condutas realizadas mediante as complicações apresentadas.

$\mathrm{Na}$ apresentação de quadro clínico com febre, deve-se fazer exame clínico da pele: observar área de inserção de cateter; hemocultura de sangue periférico e de sague do cateter; hemograma completo; bioquímica; radiografia do tórax: se houver presença de sinais e/ou sintomas de acomentimento de vias aéreas ou pulmonar; retirada de CVC na presença de infecção do tú-

FIGURA 3 - Gráfico do tempo (em dias) desde a implantação do cateter port a cath até a constatação do óbito. Hospital Federal de Bonsucesso, Rio de Janeiro, 2018.

Tempo com o cateter port a cath: da implantação ao óbito.
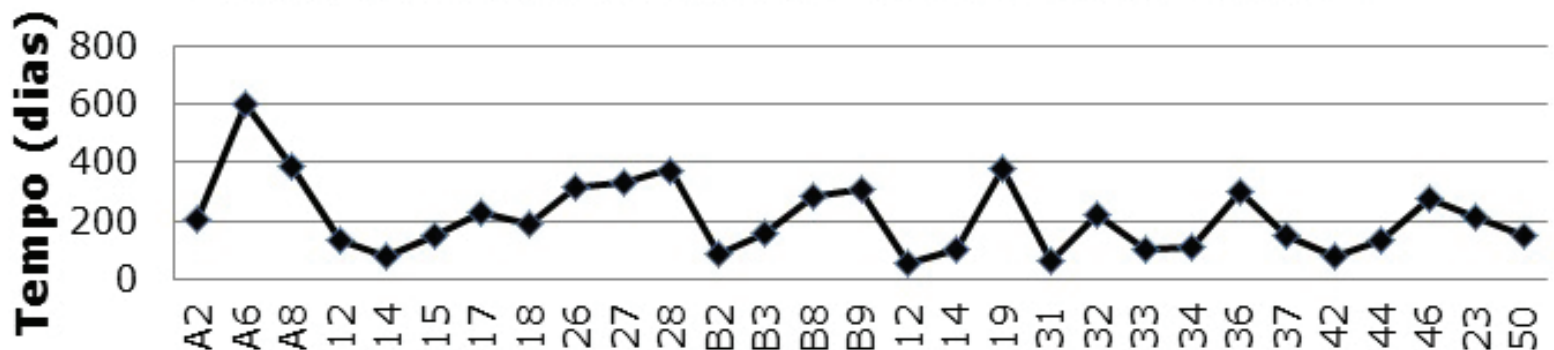

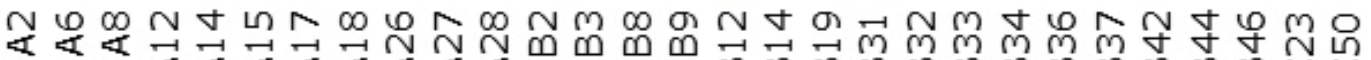

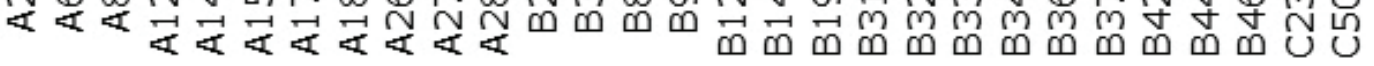


nel do cateter ou da região periport ${ }^{(15)}$. Realizar exame de urina tipo I; cultura e bacterioscopia de secreções anormais e de lesões cutâneas; coprocultura, se diarreia presente; urocultura, caso haja sinais e sintomas pertinentes ao trato urinário, presença de sonda vesical de demora e se urina I revelar anormalidades; líquor completo: se houver suspeita de acometimento neurológico e contagem de plaquetas normal(15).

Em relação a infecção do cateter, deve-se realizar antibioticoterapia venosa empírica - esquema de primeira escolha: Cefepima $2 \mathrm{~g} \mathrm{EV} \mathrm{8/8h.} \mathrm{Alternativas:} \mathrm{Ceftazidima}$ $2 \mathrm{~g} 8 / 8 \mathrm{~h}$ ou Imipenem 500mg 6/6h. Associação com:Vancomicina Ig I2//2h; agente/foco identificado, 3-5 dias de antibioticoterapia, baseando-se no antibiograma, sempre considerando que no caso de bacteremia, é fundamental o emprego de antibióticos de largo espectro; manter o antibiótico por 7 dias no mínimo, sendo necessária a recuperação clínica do paciente; persistência da febre mesmo após 3 dias de antibioticoterapia empírica: devem ser revistos: exame físico, radiografia de tórax, cateteres, culturas, nível sérico dos antibióticos e considerar exames de imagem (outros) na suspeita de abcessos; deverá ser associada Vancomicina (se não estiver no esquema empírico) e considerar a associação de Anfotericina B na dose de 0,4 a $\mathrm{Img} / \mathrm{Kg} / \mathrm{dia} \mathrm{EV}$ a cada $24 \mathrm{hs}$; remoção do cateter $^{(15)}$. Esta complicação é considerada a mais séria, devendo ser reconhecida precocemente por meio de rigorosa observação clínica, métodos diagnósticos rápidos e sensíveis e tratamento, sendo a quimioterapia suspensa até seu controle ${ }^{(16)}$.

No pneumotórax, a posição do cateter na veia cava superior deve ser confirmada por radiografia após a implantação do cateter port a cath ${ }^{(17)}$; manter a observação intra-hospitalar por 24 horas, com reavaliação clínica e radiológica neste período, confirmada a estabilidade após as 24 horas iniciais, o paciente pode ser acompanhado ambulatorialmente até a resolução completa do pneumotórax, confirmada por radiografia de tórax; com o diagnóstico de pneumotórax de grande magnitude, deve ser realizado algum procedimento invasivo que assegure a reexpansão do parênquima pulmonar colapsado: drenagem torácica ${ }^{(18)}$, onde os drenos podem ser conectados em frasco com selo d'água; a reexpansão do pulmão deve ser assegurada, caso isto não ocorra após a drenagem, a aspiração contínua controlada (com pressão negativa de até $20 \mathrm{~cm}$ de água) pode ser necessária, juntamente com a fisioterapia respiratória ${ }^{(19)}$.

No abaulamento em região infraclavicular, deve-se observar se há edema de pescoço, da garganta ou do braço; ou edema subcutâneo devido ao dano ao cateter ou ao líquido infundido retornando para fora da veia; alguns cirurgiões podem solicitar que o port não seja utilizado até que o edema pós operatório tenha diminuído(17).
Na ausência de progressão na implantação do cateter, não se deve avançar se houver resistência na implantação do cateter ${ }^{(17)}$.

No disposicionamento do cateter deve-se realizar radiografia para confirmar o posicionamento do cateter; $\mathrm{O}$ cateter deve ser estabilizado com técnica asséptica para prevenir a sua migração e preservar a integridade do dispositivo de acesso venoso ${ }^{(17)}$.

$\mathrm{Na}$ ausência de fluxo e refluxo ao puncionar cateter, a posição da agulha é verificada pelo retorno do sangue. Se não for possível a irrigação ou a aspiração, a agulha talvez precise ser empurrada mais adiante em direção ao septo ou reposicionada; Observar se há edema de pescoço, da garganta ou do braço; ou edema subcutâneo devido ao dano ao cateter ou ao líquido infundido retornando para fora da veia; Solicitar que o paciente troque de posição (por exemplo, posição de Trendelemburg) para aumentar o fluxo venoso, além de tossir ou respirar profundamente para ajudar a afastar o cateter da parede da veia;Tentar reposicionar a agulha; Não infundir quimioterapia até que a colocação da ponta seja confirmada ${ }^{(17)}$; Realizar radiografia de tórax para avaliar possível fragmentação do cateter ${ }^{(20)}$.

Em relação ao extravasamento de quimioterápicos a primeira medida a ser tomada é a interrupção da infusão, heparinização do cateter e, através do orifício da agulha, retirar por compressão o máximo de líquido possível. Reações inflamatórias e ulceração podem ocorrer até a segunda semana ${ }^{(16)}$.

\section{CONCLUSÃO}

Diante da incidência das complicações de cateter port a cath nos anos de 2014 a 2016, a infecção é a mais prevalente, sendo mencionada em 14 prontuários dos 28 que apresentaram complicações.As complicações menos citadas foram extravasamento de quimioterapia e trombose, citadas uma vez. Todas as complicações encontradas nesta pesquisa estão coerentes com os achados de estudos já existentes na temática. Em comparação com outros artigos vigentes, a infecção é a complicação que predomina-se no uso do cateter de quimioterapia em paciente oncológico.Visto que, de 2012 a 2016 houveram publicados apenas 25 artigos científicos acerca da temática, sendo em 2015 o último estudo com pesquisa documental objetivando as complicações do cateter port a cath, a pesquisa vigente proporcionará benefícios para a assistência em saúde através dos achados informados.

A equipe de saúde é a principal responsável pelas complicações do cateter port a cath, seja nas técnicas utilizadas na implantação do cateter ou no manuseio na rotina de administração de quimioterápicos e manutenção do mesmo. Cabe repensar a abordagem técnica empregada para cada ação. Para isto, são necessárias ações educativas que promo- 
vam o aperfeiçoamento das técnicas assépticas. $O$ conhecimento científico acerca de cada etapa de respectivos procedimentos não tem sucesso se após a sua implementação ocorre infecção.

Diante dos relatos encontrados na pesquisa documental, a equipe de enfermagem é a que mais evolui nos prontuários acerca da identificação do início de um processo infeccioso ou outra complicação encontrada. Entende-se que o cliente além de desenvolver vínculo pelos profissionais de enfermagem através das intensas e contínuas sessões de quimioterapia, o profissional também consegue ter um olhar holístico acerca da saúde de seu cliente através desse contato mais íntimo, permitindo observar assiduamente durante as sessões de quimioterapia o sítio de punção, assim como, os exames laboratoriais anteriores a quimioterapia.

Acreditamos que essa pesquisa possa ainda contribuir para a qualidade da assistência à saúde e a satisfação da clientela com câncer durante o tratamento e, oferecer um aporte de conhecimentos aos profissionais de enfermagem, elevando os padrões de assistência, contribuindo para a preservação da saúde no manejo clínico de cuidado com o câncer.

O impacto do resultado da pesquisa poderá promover a melhoria da assistência, maior disponibilidade para as mudanças de atitude e comportamento da equipe de enfermagem.

Sob a perspectiva do ensino da enfermagem, esperamos que essa pesquisa contribua para um maior en- tendimento acerca da temática discutida, nos preparando assim, como profissionais a desenvolver uma concepção onde muitas complicações do cateter port a cath podem ser prevenidas pelo empenho das práticas assistenciais corretas e de qualidade de toda equipe de enfermagem.

Com relação a pesquisa, acreditamos que a análise das complicações que levam a retirada do cateter port a cath no paciente oncológico, permitirá construir recursos assistenciais para os cuidados de enfermagem. Sendo assim, é de grande valia para a produção científica nessa área, colaborando no avanço das discussões e na ampliação de conhecimentos sobre a assistência oncológica. Este paciente deve ser o centro da relação do cuidado/ atenção, que deve ser integral e holístico, atendendo suas expectativas e necessidades, como um ser humano envolvido em contexto biopsicossocial.

Além disso, o desdobramento deste estudo poderá ampliar os debates sobre o tema das complicações do cateter port a cath na Unidade de Treinamento em Serviço, assim como em grupos de pesquisas que tenham interesses semelhantes. Todas as reuniões criariam incentivos para o debate desta problemática na prática de atenção oncológica, promovendo produções científicas para ampliar o conhecimento.

São limitações deste estudo, a ausência de alguns prontuários no Arquivo Médico e/ou ausência dos prontuários que ficam armazenados na Secretaria de Oncologia, impossibilitando-nos de fazer uma análise documental completa de ambos os prontuários, sendo assim, excluídos da pesquisa; e a deficiência de registro da equipe médica. 


\section{REFERÊNCIAS}

I. Bonassa EMA. Enfermagem em terapêutica oncológica. São Paulo:Atheneu; 2005.

2. Geraix APM, Campos GF, Campos RG. Cateter venoso totalmente implantável. Revista Terra e Cultura. 2007; 23 (44):44-9.

3. Brandão MA, Rodrigues Z, Sampaio S, Acioli J, Sampaio C. Cateter venoso totalmente implantável em 278 pacientes oncológicos. Rev Bras Cancerol. 2000; 46 (I): 49-56.

4. Heibl C, Trommet V, Burgstaller S, Mayrbaeurl B, Baldinger C, Koplmuller R et al. Complications associated with the use of Port-a-Caths in patients with malignant or haematological disease: a single-centre prospective analysis. Eur J Cancer Care (Engl). 2010; 19 (5): 676-8I.

5. Nishiari K, Malavolta LC, Saes GF, Langer M, Sobrinho AC, Zerati $A E$, et al. Cateteres venosos totalmente implantáveis para quimioterapia experiência em 415 pacientes.Acta Oncológica Brasileira. 2003; 23 (2): 432-40.

6. Teixeira JC, Santos CC, Pereira SR, Santos ACP. Avaliação da instalação e da utilização de sistemas para acesso venoso central totalmente implantável em mulheres com câncer. Rev Bras Mastol. 200 I; I I (4): I 32-6.

7. Liangos O, Gul A, Madias NE, Jaber BL. Long-therm management of the tunneled venous catheter. Semin Dial. 2006; 19 (2): I58-64.

8. Galdino-Júnior H, Lima BR, Santos SLV, Neves HCC,Tipple AFV. Adesão às precauções padrão durante a realização de curativos pela equipe de enfermagem. Rev Enferm Atual. [Internet]. 2018 [acesso em 29/05/20 I8];84:45-58. Disponível em https:// revistaenfermagematual.com.br/uploads/revistas/22/04.pdf

9. INSTITUTO NACIONAL DE CÂNCER JOSÉ ALENCAR GOMES DA SILVA. Estimativa 2018. Incidência de Câncer no Brasil. Rio de Janeiro: INCA, 2017. I28p. Disponível em: http://www.inca.gov.br/estimativa/20 I 8/introducao.asp. Acesso em: 29 mai. 2018.

I0. Fletcher RH, Fletcher SW,Wagner EH. Epidemiologia clínica: elementos essenciais. Porto Alegre:Artmed; 2003.

I I. Haddad N. Metodologia de estudos em ciências da saúde. São Paulo: Roca; 2004.

12.Selltiz C, Jahoda M, Deutsch M, Cook SM. Métodos de pesquisa nas relações sociais. São Paulo: Herder, 1965.

13. Minayo MCS. O desafio do conhecimento. São Paulo: HUCITEC, 2007.

14. Ferreira PL. Estatística Descritiva e Inferencial [breves notas]. Faculdade de Economia. Universidade de Coimbra. 2005; 118 p.

15. Hospital das Clínicas da Faculdade de Medicina da Universidade de São Paulo, Divisão de Oncologia - Instituto de Radiologia. Manual de Conduta do Serviço de Oncologia. São Paulo: 2003.

16. Brandão MA, Rodrigues Z, Sampaio S, Acioli J, Sampaio C. Cateter venoso totalmente implantável em 278 pacientes oncológicos. Rev Bras Cancerol. 2000;46(I):49-56.

17. Gates RA, Fink RM. Segredos em Enfermagem Oncológica. Porto Alegre:Artmed; 2009.

18. Silva FS, Campos RG. Complicações com o uso do cateter totalmente implantável em paciente oncológicos: Revisão Integrativa. Cogitare Enferm. 2009; I4(I): I59-64.

19. Filho LOA, Campos JRM, Haddad R. Pneumotórax. J Bras Pneumol. 2006; 32(4):2I2- 16.

20. Oliveira AF, Filho HO. Desconexão de cateter para quimioterapia: uma complicação rara? JVasc Bras. 20 I6; I 5(4):328-333. 